\title{
Coupled electron and phonon transport in one-dimensional atomic junctions
}

\author{
J. T. Li* and Jian-Sheng Wang⿵ \\ Center for Computational Science and Engineering and Department of Physics, \\ National University of Singapore, Singapore 117542, Republic of Singapore
}

(Dated: August 5, 2021)

\begin{abstract}
Employing the nonequilibrium Green's function method, we develop a fully quantum mechanical model to study the coupled electron-phonon transport in one-dimensional atomic junctions in the presence of a weak electron-phonon interaction. This model enables us to study the electronic and phononic transport on an equal footing. We derive the electrical and energy currents of the coupled electron-phonon system and the energy exchange between them. As an application, we study the heat dissipation in current carrying atomic junctions within the self-consistent Born approximation, which guarantees energy current conservation. We find that the inclusion of phonon transport is important in determining the heat dissipation and temperature change of the atomic junctions.

PACS numbers: 71.38.-k,63.20.Kr,72.10.Bg
\end{abstract}

\section{INTRODUCTION}

The electronic transport and phononic transport in meso- and nano-structures have attracted a great deal of interest in the past two decades, although their development is not so parallel sometimes. These structures display important quantum effects due to the confinement in one or more directions 1 . The quantized electrical conductance $^{2}$ was observed much earlier than that of the thermal conductance ${ }^{3}$ mainly due to the difficulty in measuring the thermal transport properties. Electrons and phonons are not two isolated systems. Their interactions are important for both electronic and phononic transport. With the development of both fields there arises the requirement to study the coupled electron-phonon transport from time to time. When studying electronic transport problems, one usually assumes that electrons interact with some phonon bath where the phonons are in their thermal equilibrium state characterized by the Bose distribution. This simple assumption is not able to give satisfactory results in some cases where the phonons are driven out of equilibrium by the electrons. This is especially true in places where the thermal conductance is low or the phonon relaxation is slow $\underline{4.5}$. To take into account the nonequilibrium phonon effect, one usually introduces into the electronic transport formalism some phenomenological parameters that describe the phonon relaxation process. In engineering applications, as the size of the electronic devices decreases to nanoscale, the heat dissipation and conduction in these structures become critical issues, which may influence the electronic properties dramatically $\underline{\underline{6}}$. Only studying the electronic transport is not enough in these cases. On the other hand, heat transport in one-dimensional (1D) structures has received considerable attention recently $\underline{6,7,8}$. Fourier's law of heat conduction is no longer valid in many $1 \mathrm{D}$ systems. The microscopic origins of the macroscopic Fourier's law remain one of the most frustrating problems in nonequilibrium statistical mechanics. Since the electrons and phonons both contribute to the heat conduction, their relative roles in many nanostructures are still not clear.
Especially in semiconductors, which one carries the majority of the thermal current is not a trivial problem. To answer these questions, we need some general models, which take into account the electron, phonon transport, and their mutual interactions.

Theoretically, although the development of electronic transport in 1D structures has been very striking, that of the phononic transport is relatively slow. Classical molecular dynamics (MD) and the Boltzmann-Peierls equation are the widely used methods in phononic transport. MD method is not accurate below the Debye temperature, while the Boltzmann-Peierls equation can not be used in nanostructures without translational invariance. In both cases, the quantum effect becomes important $\underline{1}$. Only recently, the nonequilibrium Green's function method $9,10,11,12$, which has been widely used to study the electronic transport, has been applied to study the quantum phononic transport13,14,15,16,17. As far as we know, the study of the coupled electronic and phononic transport in nanostructures is rare 18,19,20,21. In Ref. 19, the authors considered the nonequilibrium phonons in molecular transport junctions. Galperin and co-authors analyzed the heat generation and conduction in molecular systems $\frac{18}{}$. In this paper, using the nonequilibrium Green's function method, we study the coupled electronic and phononic transport in $1 \mathrm{D}$ atomic junctions. The formalism is similar to that of Ref. 18. In our model the electron subsystem is described by a singleorbital tight-binding Hamiltonian, and the phonon subsystem is described in a harmonic approximation. We assume that the electron-phonon interaction is weak so that the perturbative treatment is valid. The stronginteraction case is the scope of future work.

The rest of the paper is organized as follows. In Sec. II. we introduce the 1D model system, and derive expressions for the electrical, energy current of the coupled electron-phonon system. In Sec. III] we show the heat generation in one- and two-atom structures under different model parameters. Sec. IV is the conclusion. In Appendix $\mathrm{A} \| \mathrm{C}$ we give some technical details of our derivation. 


\section{COUPLED ELECTRONIC AND PHONONIC TRANSPORT}

\section{A. The Hamitonian}

Our model system is an infinite 1D atomic chain as shown in Fig. 1. The electrons and atoms are only allowed to move in the longitudinal direction. We treat the atoms as coupled harmonic oscillators, and take into account their nearest neighbour interactions up to the second order. We assume that there is only one single electronic state for each atom and take into account hopping transitions between the nearest states. This corresponds to a single-orbital tight-binding model. Also, we assume that there is only one spin state for each orbital. Following Caroli ${ }^{22}$, we divide the whole system into one central region and two semi-infinite leads, which act as electrical and thermal baths (Fig. 1). The Hamiltonian of the whole system is

$$
\begin{aligned}
H & =\sum_{\alpha=L, C, R ; \beta=\mathrm{e}, \mathrm{ph}} H_{\beta}^{\alpha} \\
& +\sum_{\alpha=L, R ; \beta=\mathrm{e}, \mathrm{ph}}\left(H_{\beta}^{\alpha C}+H_{\beta}^{C \alpha}\right)+H_{\mathrm{eph}} .
\end{aligned}
$$

The electron-phonon interaction Hamiltonian $H_{\text {eph }}$ is non-zero only in the central region. The electron Hamiltonian reads

$$
H_{\mathrm{e}}^{\alpha}=\sum_{i} \varepsilon_{i}^{\alpha} c_{i}^{\dagger \alpha} c_{i}^{\alpha}+\sum_{|i-j|=1} t_{i j}^{\alpha} c_{i}^{\dagger \alpha} c_{j}^{\alpha}
$$

where $c_{i}^{\dagger \alpha}$ and $c_{i}^{\alpha}$ are the electron creation and annihilation operators. $\varepsilon_{i}^{\alpha}$ is the electron onsite energy, and $t_{i j}^{\alpha}$ is the hopping energy between adjacent states. $i$ and $j$ run over the sites in the $\alpha$ region. The coupling Hamiltonian with the leads is

$$
H_{\mathrm{e}}^{L C}=\sum_{i j} t_{i j}^{L C} c_{i}^{\dagger L} c_{j}^{C}
$$

and

$$
H_{\mathrm{e}}^{C R}=\sum_{i j} t_{i j}^{C R} c_{i}^{\dagger C} c_{j}^{R}
$$

$H_{\mathrm{e}}^{C L}$ and $H_{\mathrm{e}}^{R C}$ have similar expressions. We also have $t^{\alpha C}=t^{C \alpha^{\dagger}}, \alpha=L, R$. For our 1D tight-binding model, $t^{\alpha C}$ has only one non-zero element. If we label the central atoms with indices 1 to $n$ as shown in Fig. 1, the non-zero elements will be $t_{01}^{L C}, t_{10}^{C L}, t_{n+1, n}^{R C}$, and $t_{n, n+1}^{C R}$.

The phonon Hamiltonian is

$$
H_{\mathrm{ph}}^{\alpha}=\frac{1}{2} \sum_{i} \dot{u}_{i}^{\alpha} \dot{u}_{i}^{\alpha}+\frac{1}{2} \sum_{|i-j|=0,1} u_{i}^{\alpha} K_{i j}^{\alpha} u_{j}^{\alpha}
$$

$u_{i}^{\alpha}$ and $\dot{u}_{i}^{\alpha}$ are the mass-renormalized atom displacement and momentum operator. $K_{i i}^{\alpha}=2 K_{0}^{\alpha} / m_{i}^{\alpha}$, and $K_{i j}^{\alpha}=$

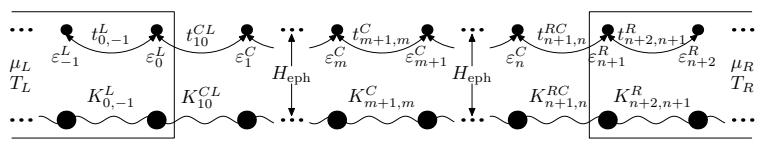

FIG. 1: Shematic diagram of the 1D coupled electron-phonon system and the parameters used in the model. The big dots in the bottom line represent atoms, while the small dots in the upper line represent electron states. They are coupled via the electron-phonon interaction.

$-K_{0}^{\alpha} / \sqrt{m_{i}^{\alpha} m_{j}^{\alpha}}(i \neq j)$. Here $K_{0}^{\alpha}$ is the spring constant, and $m_{i}^{\alpha}$ is the mass of the $i$ th atom in the $\alpha$ region. Like the electrons, the coupling Hamiltonian with the leads is

$$
H_{\mathrm{ph}}^{L C}=\frac{1}{2} \sum_{i j} u_{i}^{L} K_{i j}^{L C} u_{j}^{C},
$$

and

$$
H_{\mathrm{ph}}^{C R}=\frac{1}{2} \sum_{i j} u_{i}^{C} K_{i j}^{C R} u_{j}^{R}
$$

We also have $K^{C \alpha}=K^{\alpha C^{\dagger}}$. The non-zero elements are $K_{01}^{L C}, K_{10}^{C L}, K_{n+1, n}^{R C}$, and $K_{n, n+1}^{C R}$.

The electron-phonon interaction is included within the adiabatic Born-Oppenheimer approximation. First, the electron subsystem is solved with all the atoms in their equilibrium positions. Then, the isolated phonon subsystem is considered. After that, the electron-phonon interaction is turned on by allowing the atoms to oscillate around their equilibrium positions. Within this picture, the electron-phonon interaction is 11

$$
H_{\mathrm{eph}}=\sum_{i, j, k} M_{i j}^{k} c_{i}^{\dagger} c_{j} u_{k} .
$$

The interaction matrix element is $M_{i j}^{k}=\left\langle i\left|\frac{\partial H_{\mathrm{e}}}{\partial u_{k}}\right| j\right\rangle$. All the operators in Eq. (8) are in the central region, so we omitted the superscript $C$. In our model, the electron operators are in the second quantization, while that of the phonons are in the first quantization.

\section{B. Green's functions}

The nonequilibrium Green's function method for the electronic transport is discussed in Refs. 9, 10,11,12, and that for the phononic transport in Refs. 13, 14, 15, 16. Here we concentrate on the electron-phonon interactions. The definition of the electron contour-ordered Green's function is $G_{j k}\left(\tau, \tau^{\prime}\right)=-i\left\langle\mathcal{T}\left\{c_{j}(\tau) c_{k}^{\dagger}\left(\tau^{\prime}\right)\right\}\right\rangle$, and the phonon counterpart is $D_{j k}\left(\tau, \tau^{\prime}\right)=-i\left\langle\mathcal{T}\left\{u_{j}(\tau) u_{k}\left(\tau^{\prime}\right)\right\}\right\rangle$. Here $\tau$ is time on the Keldysh contour, and $\mathcal{T}\{\cdots\}$ is the contour-ordered operator. We set $\hbar=1$ throughout the formulas. Without the electron-phonon interaction, the isolated electron and phonon problem can be solved exactly. We denote these Green's functions 
as $G_{0}\left(\tau, \tau^{\prime}\right)$ and $D_{0}\left(\tau, \tau^{\prime}\right)$, respectively. In our case, it is convenient to write the Hamiltonians as matrices and work in the energy space. The electron retarded and advanced Green's functions are $G_{0}^{r}(\varepsilon)=$ $G_{0}^{a \dagger}(\varepsilon)=\left[(\varepsilon+i \eta) I-H_{\mathrm{e}}^{C}-\Sigma_{L}^{r}(\varepsilon)-\Sigma_{R}^{r}(\varepsilon)\right]^{-1} . \quad I$ is an identity matrix, and $\eta \rightarrow 0^{+}$. The retarded selfenergy $\Sigma_{\alpha}^{r}=t^{C \alpha} g_{\alpha}^{r} t^{\alpha C}$ is due to the interactions with the lead $\alpha$. The retarded Green's function of the semiinfinite lead $g_{\alpha}^{r}$ can be obtained analytically (Appendix A). The "less than" Green's function is given by $G_{0}^{<}=$ $G_{0}^{r}\left(\Sigma_{L}^{<}+\Sigma_{R}^{<}\right) G_{0}^{a}$, where $\Sigma_{\alpha}^{<}=-f_{\alpha}^{\mathrm{e}}\left(\Sigma_{\alpha}^{r}-\Sigma_{\alpha}^{a}\right)$. $f_{\alpha}^{\mathrm{e}}$ is the Fermi-Dirac distribution. The phonon retarded and advanced Green's functions are $\underline{\underline{23}} D_{0}^{r}(\omega)=D_{0}^{a \dagger}(\omega)=$ $\left[(\omega+i \eta)^{2} I-K^{C}-\Pi_{L}^{r}(\omega)-\Pi_{R}^{r}(\omega)\right]^{-1}$. The lead retarded self-energy is $\Pi_{\alpha}^{r}(\omega)=K^{C \alpha} d_{\alpha}^{r}(\omega) K^{\alpha C} . d_{\alpha}^{r}$ also has analytical expression (Appendix $\underline{A}$ ). The phonon "less than" Green's function is $D_{0}^{<}=D_{0}^{r}\left(\Pi_{L}^{<}+\Pi_{R}^{<}\right) D_{0}^{a}$, where $\Pi_{\alpha}^{<}=f_{\alpha}^{\mathrm{ph}}\left(\Pi_{\alpha}^{r}-\Pi_{\alpha}^{a}\right) . f_{\alpha}^{\mathrm{ph}}$ is the Bose distribution function.

Knowing the bare electron and phonon Green's functions $G_{0}$ and $D_{0}$, we can include their interaction as perturbation. Following the standard procedure of nonequilibrium Green's function method, we can express this interaction as self-energies. The full Green's functions are obtained from the Dyson equation, e.g., for electrons $G^{r, a}=G_{0}^{r, a}+G_{0}^{r, a} \Sigma_{\text {eph }}^{r, a} G^{r, a}$, and $G^{<}=G^{r} \Sigma_{\mathrm{t}}^{<} G^{a}$. $\Sigma_{\mathrm{t}}^{<}=\Sigma_{\mathrm{eph}}^{<}+\Sigma_{\mathrm{L}}^{<}+\Sigma_{\mathrm{R}}^{<}$is the total self-energy. Keeping the lowest non-zero order (the second order) of the selfenergies, we have two (Hartree- and Fock-like) terms for the electrons, and one polarization term for the phonons. This is the so-called Born approximation $(\mathrm{BA}) \underline{11}$. The Fock self-energies are

$$
\Sigma_{m n}^{F,<}(\varepsilon)=i M_{m i}^{k} \int G_{0}^{<}{ }_{i j}(\varepsilon-\omega) D_{0 k l}^{<}(\omega) \frac{d \omega}{2 \pi} M_{j n}^{l},
$$

and

$$
\begin{aligned}
\Sigma_{m n}^{F, r}(\varepsilon)= & i M_{m i}^{k} \int \frac{d \omega}{2 \pi}\left[G_{0 i j}^{r}(\varepsilon-\omega) D_{0 k l}^{<}(\omega)\right. \\
& +G_{0}^{<}{ }_{i j}(\varepsilon-\omega) D_{0 k l}^{r}(\omega) \\
& \left.+G_{0 i j}^{r}(\varepsilon-\omega) D_{0 k l}^{r}(\omega)\right] M_{j n}^{l} .
\end{aligned}
$$

The "less than" Hartree self-energy is zero, and the retarded one is

$$
\Sigma_{m n}^{H, r}=-i M_{m n}^{i} D_{0 i j}^{r}\left(\omega^{\prime}=0\right) M_{k l}^{j} \int G_{0 l k}^{<}(\varepsilon) \frac{d \varepsilon}{2 \pi} .
$$

This term is a constant for all energies, which represents a static potential due to the presence of phonons. The self-energies for the phonons are

$$
\Pi_{m n}^{<}(\omega)=-i M_{l k}^{m} \int \frac{d \varepsilon}{2 \pi} G_{0 k i}^{<}(\varepsilon) G_{0 j l}^{>}(\varepsilon-\omega) M_{i j}^{n},
$$

and

$$
\begin{aligned}
\Pi_{m n}^{r}(\omega)= & -i M_{l k}^{m} \int \frac{d \varepsilon}{2 \pi}\left[G_{0 k i}^{r}(\varepsilon) G_{0 j l}^{<}(\varepsilon-\omega)\right. \\
& \left.+G_{0 k i}^{<}(\varepsilon) G_{0 j l}^{a}(\varepsilon-\omega)\right] M_{i j}^{n} .
\end{aligned}
$$

In Eqs. (9) 13), sum over internal indices is assumed. The self-consistent Born approximation (SCBA) is obtained by replacing all the bare Green's functions $G_{0}$ and $D_{0}$ in Eqs. (9-13) with the full $G$ and $D^{11}$. In Appendix B. we show that the SCBA fulfills the electrical and energy current conservation, while BA fails.

\section{The electrical and energy current}

The electrical and energy current can be expressed by the Green's functions. The electrical current out of the lead $\alpha$ is 11,24

$$
J_{\alpha}=e \int \frac{d \varepsilon}{2 \pi} \operatorname{Tr}\left\{G^{>}(\varepsilon) \Sigma_{\alpha}^{<}(\varepsilon)-G^{<}(\varepsilon) \Sigma_{\alpha}^{>}(\varepsilon)\right\} .
$$

The electron energy current is

$$
J_{\alpha}^{\mathrm{E}, \mathrm{e}}=\int \frac{d \varepsilon}{2 \pi} \varepsilon \operatorname{Tr}\left\{G^{>}(\varepsilon) \Sigma_{\alpha}^{<}(\varepsilon)-G^{<}(\varepsilon) \Sigma_{\alpha}^{>}(\varepsilon)\right\} .
$$

The electron heat current is obtained from Eqs. (14) 15) as $J_{\alpha}^{\mathrm{h}, \mathrm{e}}=J_{\alpha}^{\mathrm{E}, \mathrm{e}}-\mu_{\alpha} J_{\alpha} / e . \quad \mu_{\alpha}$ is the lead chemical potential. The derivation of the phonon energy current runs parallel with that of the electrons 17

$$
J_{\alpha}^{\mathrm{E}, \mathrm{ph}}=-\int \frac{d \omega}{4 \pi} \omega \operatorname{Tr}\left\{D^{>}(\omega) \Pi_{\alpha}^{<}(\omega)-D^{<}(\omega) \Pi_{\alpha}^{>}(\omega)\right\} .
$$

For phonons the energy current is the same as the heat current. When there is no electron-phonon interaction, the electron energy current is conserved throughout the structure. So is the phonon energy current. In the presence of such an interaction, only the total energy current is conserved due to the energy exchange between them. The phonons do not carry charges, so in both cases the electrical current is conserved. Since we can't get the exact self-energies in most cases, we need some approximations. Properly defined self-energies should fulfill the electrical and energy current conservation

$$
\begin{gathered}
\sum_{\alpha} J_{\alpha}=0, \\
\sum_{\alpha}\left(J_{\alpha}^{\mathrm{E}, \mathrm{e}}+J_{\alpha}^{\mathrm{E}, \mathrm{ph}}\right)=0,
\end{gathered}
$$

where $\alpha$ runs over all the leads. We justify that the SCBA fulfills these conservation laws, while the BA fails to conserve the energy current (Appendix B). Provided we satisfy these conservation laws, we can write the electrical and energy current in symmetric forms. The electrical current is

$$
J=e \int \frac{d \varepsilon}{2 \pi} \tilde{T}^{\mathrm{e}}(\varepsilon)\left[f_{L}^{\mathrm{e}}(\varepsilon)-f_{R}^{\mathrm{e}}(\varepsilon)\right] .
$$

The transmission coefficient reads

$$
\begin{aligned}
\tilde{T}^{\mathrm{e}}= & \operatorname{Tr}\left\{\frac { 1 } { 2 } \left[G^{r}\left(\Gamma_{L}+\frac{1}{2} \Gamma_{\mathrm{eph}}-S^{\mathrm{e}}\right) G^{a} \Gamma_{R}\right.\right. \\
& \left.\left.+G^{r} \Gamma_{L} G^{a}\left(\Gamma_{R}+\frac{1}{2} \Gamma_{\mathrm{eph}}+S^{\mathrm{e}}\right)\right]\right\},
\end{aligned}
$$


where $S^{\mathrm{e}}$ is

$$
S^{\mathrm{e}}=\frac{\frac{1}{2}\left(f_{R}^{\mathrm{e}}+f_{L}^{\mathrm{e}}\right) \Gamma_{\mathrm{eph}}+i \Sigma_{\mathrm{eph}}^{<}}{f_{L}^{\mathrm{e}}-f_{R}^{\mathrm{e}}} .
$$

$\Gamma_{\alpha}=i\left(\Sigma_{\alpha}^{r}-\Sigma_{\alpha}^{a}\right), \quad \alpha=L, R$ is the electron level-width function. $\Gamma_{\mathrm{eph}}=i\left(\Sigma_{\mathrm{eph}}^{r}-\Sigma_{\mathrm{eph}}^{a}\right)$ is due to the electronphonon interaction. The total energy current is

$$
\begin{aligned}
J^{\mathrm{E}}= & \int \frac{d \varepsilon}{2 \pi} \varepsilon\left\{\tilde{T}^{\mathrm{e}}(\varepsilon)\left[f_{L}^{\mathrm{e}}(\varepsilon)-f_{R}^{\mathrm{e}}(\varepsilon)\right]\right. \\
& \left.-\frac{1}{2} \tilde{T}^{\mathrm{ph}}(\varepsilon)\left[f_{L}^{\mathrm{ph}}(\varepsilon)-f_{R}^{\mathrm{ph}}(\varepsilon)\right]\right\} .
\end{aligned}
$$

The phonon transmission coefficient is

$$
\begin{aligned}
\tilde{T}^{\mathrm{ph}}= & \operatorname{Tr}\left\{\frac { 1 } { 2 } \left[D^{r}\left(\Lambda_{L}+\frac{1}{2} \Lambda_{\mathrm{eph}}-S^{\mathrm{ph}}\right) D^{a} \Lambda_{R}\right.\right. \\
& \left.\left.+D^{r} \Lambda_{L} D^{a}\left(\Lambda_{R}+\frac{1}{2} \Lambda_{\mathrm{eph}}+S^{\mathrm{ph}}\right)\right]\right\},
\end{aligned}
$$

where $S^{\mathrm{ph}}$ is

$$
S^{\mathrm{ph}}=\frac{\frac{1}{2}\left(f_{R}^{\mathrm{ph}}+f_{L}^{\mathrm{ph}}\right) \Lambda_{\mathrm{eph}}-i \Pi_{\mathrm{eph}}^{<}}{f_{L}^{\mathrm{ph}}-f_{R}^{\mathrm{ph}}} .
$$

$\Lambda_{\alpha}=i\left(\Pi_{\alpha}^{r}-\Pi_{\alpha}^{a}\right)$ is the phonon level-width function. $\Lambda_{\mathrm{eph}}=i\left(\Pi_{\mathrm{eph}}^{r}-\Pi_{\mathrm{eph}}^{a}\right)$ is due to the electron-phonon interaction. Eqs. (19,24) are the generalization of the Caroli formula 22 to include the electron-phonon interaction.

\section{HEAT GENERATION IN CURRENT CARRYING 1D ATOMIC JUNCTIONS}

As an application of the formalism in Sec. III, we study the heat dissipation in current-carrying $1 \mathrm{D}$ atomic junctions $18,25,26,27,28,29,30,31$. In the presence of potential difference between the two leads, there will be electrical current flowing between them. When the electrons pass the central region, there is energy exchange between the electron and phonon systems. The energy dissipated into the phonon system makes the atom temperature higher than that of the leads if it is not efficiently conducted to the leads. If the electron-phonon interaction is weak, the energy dissipated into the phonon system is only a small fraction of the electron energy current. But this small fraction still influences the transport properties of the atomic junction and even leads to junction breakup 32,33 , especially when the thermal conductance is low. Different models have been used to study the local heating effect. Some simply assume that the phonons are in their thermal equilibrium states ${ }^{31}$. Some take into account the phonon transport by using the rate equations ${ }^{30}$ or other semi-classical models $26,27,32$. Few of them take into account the quantum effect in heat transport 18,34 . Our model treats the electron and phonon transport on an equal quantum-mechanical footing, and includes their interactions self-consistently. The heat generation is given by (Eq. (B9))

$$
\begin{aligned}
Q= & i \int \frac{d \varepsilon}{2 \pi} \int \frac{d \omega}{2 \pi} \omega \\
& \times\left[G_{n m}^{>}(\varepsilon) M_{m i}^{k} D_{k l}^{<}(\omega) G_{i j}^{<}(\varepsilon-\omega) M_{j n}^{l}\right] .
\end{aligned}
$$

At zero temperature, we can get an analytical expression Eq. (C1) for a single-atom structure by using the bare Green's functions $G_{0}$ and $D_{0}$ in Eq. (25) (Appendix C). Equation (C1) can reproduce most qualitative features of heat generation in a single atom, except that it does not take into account heat conduction in the phonon system.

We first study the case where the lead energy band is wide compared to the voltage applied to the structure. For most metallic leads, this condition should hold. In the weak electron-phonon coupling regime, the Born approximation should give acceptable results for the heat generation, although physically it is not a good approximation. Figure 2 shows the heat generation of a single atom ( $n=1$ in Fig. 1) computed using Eqs. (B5) and (B8) under BA and SCBA, respectively. The parameters used in the calculation are stated in the figure caption. With these parameters, the electron energy band is in the range $-1 \leq \varepsilon \leq 1 \mathrm{eV}$. The chemical potential of each lead is zero in equilibrium. The phonon energy is approximately $\omega=0.05 \mathrm{eV}$. In all the results presented in this section, the temperature is $T=4.2 \mathrm{~K}$, the electronphonon coupling matrix $M=0.08 \mathrm{eV} /\left(\AA \cdot \mathrm{amu}^{\frac{1}{2}}\right)$. The cut-off energy of the electron system is $2.1 \mathrm{eV}$, and the phonon system is $0.2 \mathrm{eV}$. The energy spacing is discretized into grids of $1 \mathrm{meV}$. Equation (B5) gives the energy decrease of the electron system, while Eq. (B8) gives the energy increase of the phonon system. Numerical results from Eq. (B5) and Eq. (B8) under SCBA have some slight discrepancy. This is due to numerical inaccuracies. But most of the discrepancy under BA comes from the difference between the bare and the full Green's functions, which may become even larger for some parameters. So BA should be used with care in the study the energy exchange between the electron and phonon system. We also note that although Eqs. (B5) and (B7) are equivalent, numerical result from Eq. ( $\overline{\mathrm{B} 5}$ ) is unstable in many cases. The reason is that the energy exchange between the electron and the phonon system is only a small fraction of the total electrical energy current. Equation (B5) is the difference between two large numbers, so our numerical integration has to be accurate enough to get a reasonable result ${ }^{30}$. On the contrary, Eq. (25) is much more stable since we have got the difference analytically. All the results presented below use this equation.

From Fig. 2, we can see two threshold values in heat generation. The first one corresponds to the onset of phonon emission. Under low temperatures, the equilibrium phonon occupation is very small, so the phonon absorption process seldom takes place. If the applied bias is smaller than the phonon energy, electrons don't 


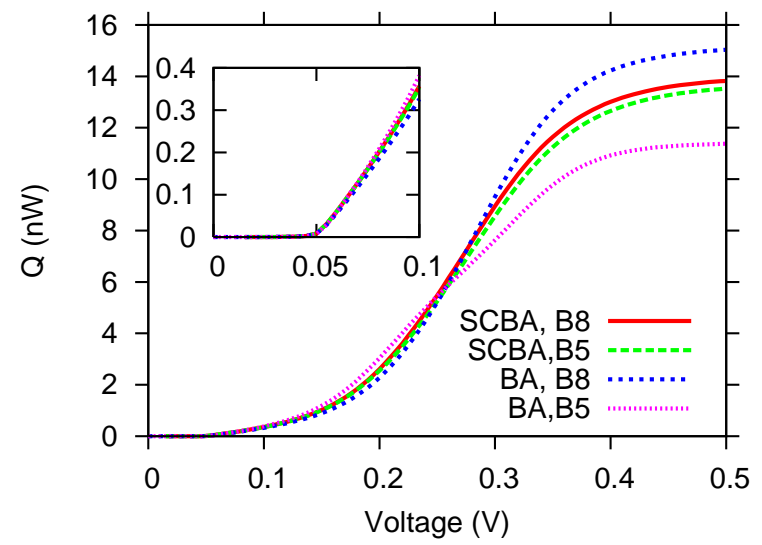

FIG. 2: Comparison of different methods to compute the heat generation in a single atom structure. The four curves correspond to results from Eqs. (B5) and (B8) under BA and SCBA, respectively. If we label this single atom as index 1, its electronic onsite energy is written as $\varepsilon_{1}^{C}=0.1 \mathrm{eV}$. The onsite energy of the leads is $\varepsilon^{L}=\varepsilon^{R}=0 \mathrm{eV}$. The hopping energy is $t_{i j}^{L}=t_{i j}^{R}=0.5 \mathrm{eV}$. The non-zero electronic coupling with the lead is $t_{10}^{C L}=t_{21}^{R C}=0.1 \mathrm{eV}$. The matrix element of the single atom is $K_{11}^{C}=0.654 \mathrm{eV} /\left(\AA^{2}\right.$. amu). The spring constant between the lead atoms is $K_{i j}^{L}=K_{i j}^{R}=0.654$ $\mathrm{eV} /\left(\AA^{2} \cdot \mathrm{amu}\right)$. The non-zero atomic coupling with the leads is $K_{10}^{C L}=K_{21}^{R C}=0.127 \mathrm{eV} /\left(\AA^{2} \cdot \mathrm{amu}\right)$.

have enough energy to emit one phonon. So the heat generation is zero. Once the applied voltage is larger than the phonon energy, phonon emission turns on. The heat generation increases almost linearly with the applied bias (inset of Fig. 21). This is different from the electrical current, which increases smoothly in this regime. The second threshold value corresponds to the alignment of the left lead chemical potential with the electron onsite energy $\mathrm{eV}=2 \varepsilon_{0}$ (positive bias $\mu_{l}>\mu_{r}$ ). The electron transmission is nearly unity above the onsite energy. The larger the transmission, the larger the current and the heat generation provided that the other parameters remain unchanged. These two threshold behaviours may become less obvious when the coupling with the leads get stronger. As a result of coupling, the discrete electron and phonon density of states (DoS) extends to a small energy region around their discrete values. The continuous phonon DoS leads to the broadening of the first threshold behaviour, while the continuous electron DoS is responsible for that of the second. It is smoothed out when the coupling is large enough (Fig. 3). Only electrons whose energies are within the broadened energy spectrum can tunnel across the central atom. The heat generation reaches maximum when the electron states in one lead are all occupied in this energy range, while those in the other are all empty.

The electron-lead coupling not only leads to the electron level broadening, but it also influences the electron tunneling time. The larger this coupling, the less time electrons spend in the central region. In Fig. 3 we show the heat generation and the atom temperature for a single-atom structure under different electronic coupling strengths. The definition of temperature is ambiguous in nanostructures ${ }^{6}$. Here we use the method proposed in Ref. 18. We can only see one threshold behaviour at about $0.2 \mathrm{~V}$, which is smoothed out when the coupling is larger than $0.2 \mathrm{eV}$. The temperature and the heat generation show similar trends. The saturate voltage of heat generation increases with the strengthen of the electronlead coupling. This is due to the coupling induced atomic level broadening. The decrease of the heat generation and temperature with increasing electron-leads coupling can be easily understood. The larger this coupling, the less time electrons spend at the central atom. Since the electron-phonon interaction takes place there, the heat generation decreases. We also show the heat generation as a function of electron-lead coupling in the inset of the lower panel. The applied voltage is $0.3 \mathrm{~V}$. On one side, when the coupling is too small, few electrons can tunneling through the atom. The heat generation is small. On the other, when the coupling is very large, the electron tunneling process is too quick for the phonons to interact with the electrons. The heat generation is also small. It has a maximum value at some moderate coupling strength. This is different from the electrical current, which increases monotonously with the increase of coupling strength.

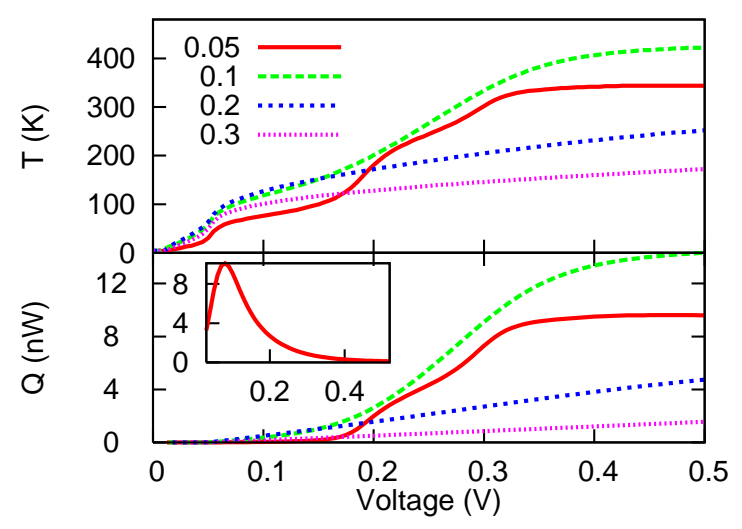

FIG. 3: Heat generation $Q$ and the atom temperature $T$ under different electron coupling strength $t_{10}^{C L}=t_{21}^{R C}=0.05,0.1$, 0.2 , and $0.3 \mathrm{eV}$, respectively. Other parameters are the same with Fig. 2 The inset shows the heat generation as a function of electron coupling strength at an applied bias $V=0.3 \mathrm{~V}$.

The atom-lead coupling determines how well the generated heat can be conducted into the surrounding leads. One of the important reasons why we are interested in the heat generation in nanostructures is that it may leads to temperature increase and even structure breakup. To study the temperature change, we need to take into account not only the heat generation, but also the heat conduction into the leads. In the simplest one-atom structure, the heat conductance is mainly determined by the atom-lead coupling. Our model includes this intrinsically. Figure 4 shows the heat generation and the atom temper- 
ature as a function of atom-lead coupling. For the heat generation, the BA and SCBA results show large difference around the resonant position, which corresponds to a perfect atomic junction. For the atom temperature, BA and SCBA give almost the same results. In the case of a perfect junction, the heat generation reaches its maximum value, while the atom temperature is the lowest. The reason is that the perfect junction has the best heat conductance. When the atom-lead coupling is weak, the heat generation is small. But the poor heat conductance can still result in a much higher temperature than the surrounding leads. We also show the heat conductance as a function of atom-lead coupling in the inset of the upper panel, which shows a sharp peak at resonance.

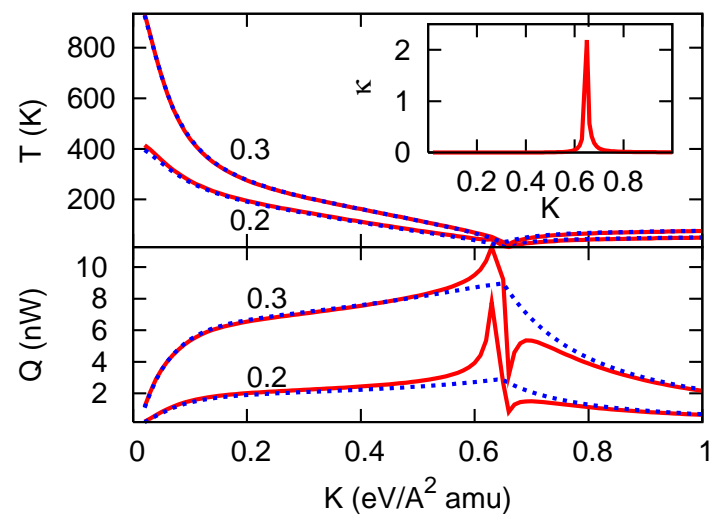

FIG. 4: Heat generation $Q$ and the atom temperature $T$ as a function of the atom-lead coupling $K_{10}^{C L}=K_{21}^{R C}=K$. Dashed and dotted lines correspond to results of SCBA and BA, respectively. Other parameters are the same with Fig. 2 The inset shows the thermal conductance $\kappa$ as a function of $K$. The unit is $1 \times 10^{-12} \mathrm{~W} / \mathrm{K}$.

In Fig. 5, we show the heat generation as a function of electron onsite energy at different biases. We assume that we can tune the the onsite energy via a gate voltage. When the applied bias is less than the phonon energy, there will be no heat generation. When the bias energy is slightly larger than the phonon energy and less than $2 \omega$, there are two energy positions where the heat generation is the largest. These two peaks are approximately at $-0.5 \mathrm{eV}+\omega$ and $0.5 \mathrm{eV}-\omega$. They merge into a single one at a bias of $e V=2 \omega$ until it reaches saturation. After that, this peak broadens, and becomes ladders. All these behaviour can also be explained by the analytical result of Eq. (C1).

In Fig. 6 we show the heat generation of a two-atom structure $(n=2$ in Fig. 11). The central region has two identical atoms. Interaction between them leads to two discrete energy levels. One is at $0 \mathrm{eV}$, and the other at $0.4 \mathrm{eV}$. When the electrical coupling between the leads and the central region is small $(0.1 \mathrm{eV})$, additional to the threshold behaviour at $e V=\omega$, there are two ladders corresponding to the phonon assisted resonant tunneling across the two electrical levels. If the electrical coupling

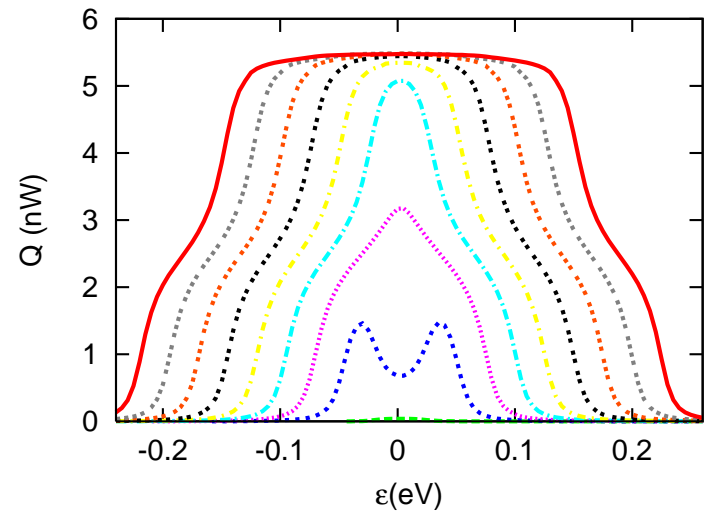

FIG. 5: Heat generation $Q$ as a function of electrical onsite energy $\varepsilon_{1}^{C}$ under different biases. From the inner to the outer side, the applied biases are $V=0.05,0.10,0.15,0.20,0.25$, $0.30,0.35,0.40,0.45$, and $0.50 \mathrm{~V}$, respectively.

gets larger $(0.2 \mathrm{eV})$, the two ladders broaden out. Again this is attributed to the coupling induced level broadening. The heat generation for the two-atom structure is much larger than that of a single-atom structure. The more the electrical levels, the larger the electrical current and heat generation. It is worth noting that for multiatom structures the distribution of the electrostatic potential may influence the results significantly 35 . In the above calculation, we assume that the two electrical levels don't change with the applied bias, and that we can tune their positions via a gate voltage.

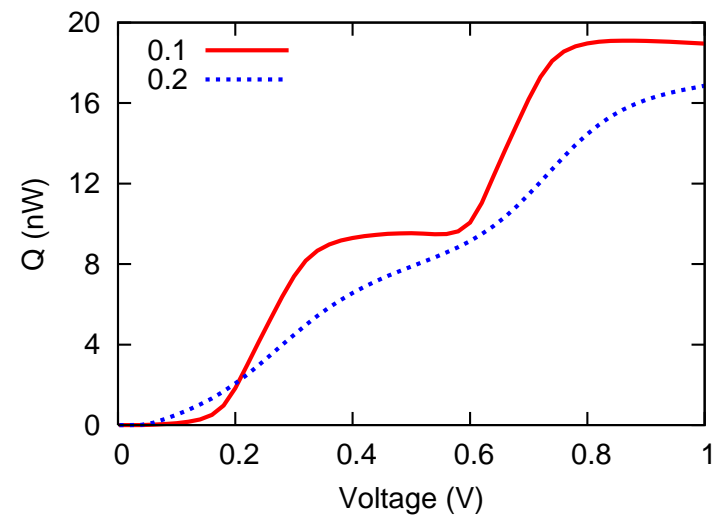

FIG. 6: Heat generation $Q$ as a function of applied voltage for a two-atom structure. The two-atom onsite energy is $\varepsilon^{C}=0.2$ $\mathrm{eV}$, the hopping energy is $t^{C}=0.1 \mathrm{eV}$, and the spring constant is $K^{C}=0.654 \mathrm{eV} /\left(\AA^{2} \cdot \mathrm{amu}\right)$. The two leads are identical. The electron onsite energy is $\varepsilon^{L}=\varepsilon^{R}=0$, and the hopping energy is $t^{L}=t^{R}=0.5 \mathrm{eV}$. Their spring constants are the same as the central region. The non-zero coupling couplings with the leads are $K_{10}^{C L}=K_{32}^{R C}=0.327 \mathrm{eV} /\left(\AA^{2} \cdot \mathrm{amu}\right)$, and $t_{10}^{C L}=t_{32}^{R C}=0.1$ (solid), $0.2 \mathrm{eV}$ (dashed), respectively.

If one of the metallic leads is replaced by a semiconductor, there will be some new features in the electrical current and the heat generation. In our simple model, 
we can alternate the electron onsite energies between two values to mimic a simple semiconductor (Appendix A). In Fig. 7 we show the heat generation and the electrical current for such kind of structure. The alternating onsite energies of the left lead are -0.1 and $-0.2 \mathrm{eV}$, respectively. This produces an energy band-gap of $0.1 \mathrm{eV}$. Other parameters are given in the figure caption. We can see that there appears negative differential conductivity in the current-voltage characteristics due to the semiconductor band-gap. This qualitatively agrees with the experimental ${ }^{36}$ and first-principle ${ }^{37}$ studies. The heat generation curve is slightly different. Additional to its threshold behaviour, the peak and valley positions are also different. The electrical current has a peak when the chemical potential of the lead is aligned with the central electrical level, while the peak of the heat generation shifts to the right by one phonon energy. This corresponds to the phonon-assisted resonant tunneling. The current and heat generation decrease when the single electrical level is within the band-gap of the left lead. The peak-to-valley ratio depends on the coupling with the semiconductor lead. In the limit of small band-gap and large coupling, we recover the metallic lead results.

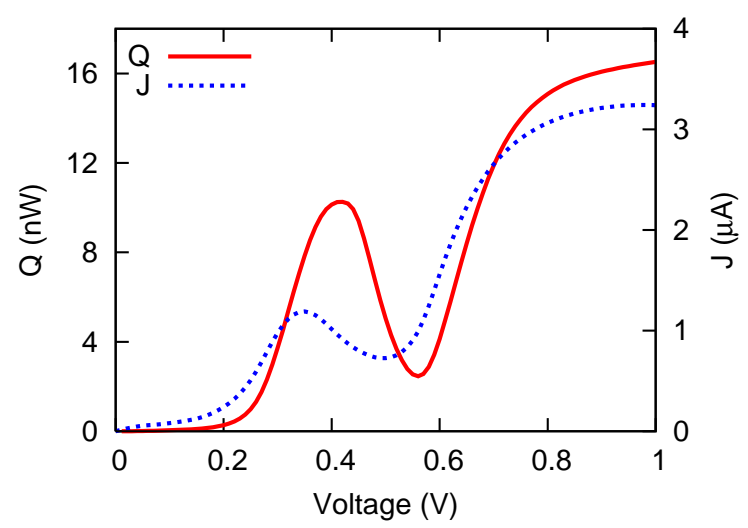

FIG. 7: Heat generation $Q$ and electrical current $J$ as a function of applied voltage for a single-atom structure. The left lead is a semiconductor. Its alternating onsite energies are -0.2 and $-0.1 \mathrm{eV}$, respectively. The chemical potential is $\mu^{L}=0.05 \mathrm{eV}$ higher than the conduction band bottom, which corresponds to $n$-type doping. Other parameters are $K_{10}^{C L}=K_{21}^{R C}=0.4 \mathrm{eV} /\left(\AA^{2} \cdot \mathrm{amu}\right), t_{10}^{C L}=t_{21}^{R C}=0.1 \mathrm{eV}$, $\varepsilon_{1}^{C}=0.1 \mathrm{eV}, K_{11}^{C}=0.654 \mathrm{eV} /\left(\AA^{2} \cdot \mathrm{amu}\right), \varepsilon^{R}=-0.05 \mathrm{eV}$, $t^{L}=t^{R}=0.5 \mathrm{eV}$, and $K^{L}=K^{R}=0.654 \mathrm{eV} /\left(\AA^{2} \cdot \mathrm{amu}\right)$.

\section{CONCLUSION}

We studied the coupled electron and phonon transport in $1 \mathrm{D}$ atomic junctions in the weak electron-phonon interaction regime. Base on the nonequilibrium Green's function method, we derived the electrical, energy current of the coupled electron-phonon system, and the energy exchange between them. We showed that the SCBA conserves the energy current. Using this formalism, we stud- ied the heat generation in one- and two-atom structures coupling with different leads under a broad range of parameters. Especially, we studied the influence of the thermal transport properties on the heat generation and atom temperature of the central region. The results on semiconductor leads agree qualitatively with the experimental and first-principle studies. This model can be easily extended to study more realistic structures such as molecular transport junctions and metallic nanowires. The electron, phonon Hamiltonian, their interaction and leadcoupling matrices can all be obtained from first-principle calculations $17,38,39$. The surface Green's functions for bulk leads can be computed by recursive method ${ }^{17,38}$. It is also possible to include the electron-electron and the phonon-phonon interactions ${ }^{14,17}$.

\section{Acknowledgments}

We thank Baowen Li, Sai Kong Chin, Jian Wang, and Nan Zeng for discussions. This work is supported in part by a Faculty Research Grant of the National University of Singapore.

\section{APPENDIX A: SURFACE GREEN'S FUNCTIONS OF THE 1D LEAD}

In this Appendix, we show that for the 1D tightbinding model the lead self-energies can be expressed analytically 17 . The electron and phonon self-energies are similar in their form. Here we take electrons as an example, and give the phonon results directly. We assume that the onsite energies of the electrons alternate between $\varepsilon_{1}$ and $\varepsilon_{2}$. The hopping energy is $t_{i j}^{\alpha}=t_{0}$. If $\varepsilon_{1}=\varepsilon_{2}$, we get a continuum band. This corresponds to a metallic lead. If they are not equal, we get two bands with a band gap. We can take the lower as the valence band (VB), and the upper as the conduction band (CB). We use this method to mimic a semiconductor lead. In this case, the semiinfinite lead has two electron states in each period. In the tight-binding model, only the left- (right-) most state of the central region is coupled to the left (right) lead. So we only need to know the surface Green's function, e.g., for the left lead it is $g_{0}=g_{00}^{r}$. We assume the retarded Green's function is

$$
g_{i j}^{r}= \begin{cases}c_{1} \lambda^{i-j} & \text { state } 1 \\ c_{2} \lambda^{i-j} & \text { state } 2 .\end{cases}
$$

Putting it into the definition of the retarded Green's functions $[(\varepsilon+i \eta) I-H] g^{r}=I$, we have

$$
\begin{array}{r}
-t_{0} c_{1}+\left(\varepsilon+i \eta-\varepsilon_{2}\right) c_{2}-t_{0} c_{1} \lambda=0, \\
-t_{0} c_{2}+\left(\varepsilon+i \eta-\varepsilon_{1}\right) c_{1} \lambda-t_{0} c_{2} \lambda=0 .
\end{array}
$$

From Eqs. (A2), we get an equation for $\lambda$

$$
\lambda^{2}+\left[2-\frac{\left(\varepsilon+i \eta-\varepsilon_{1}\right)\left(\varepsilon+i \eta-\varepsilon_{2}\right)}{t_{0}^{2}}\right] \lambda+1=0 .
$$


The condition that Eq. (A4) has travelling wave solutions gives the dispersion relation

$$
\begin{array}{ll}
\frac{\left(\varepsilon_{1}+\varepsilon_{2}\right)-\sqrt{\left(\varepsilon_{1}-\varepsilon_{2}\right)^{2}+16 t_{0}^{2}}}{2} \leq \varepsilon \leq \varepsilon_{1} & (\mathrm{VB}) \\
\varepsilon_{2} \leq \varepsilon \leq \frac{\left(\varepsilon_{1}+\varepsilon_{2}\right)+\sqrt{\left(\varepsilon_{1}-\varepsilon_{2}\right)^{2}+16 t_{0}^{2}}}{2} & (\mathrm{CB})
\end{array}
$$

We assume $\varepsilon_{1} \leq \varepsilon_{2}$ without loss of generality. The energy band-gap is $\varepsilon_{2}-\varepsilon_{1}$. If they are equal, the two bands merge into one, which corresponds to a metallic lead.

For the surface Green's function of the left lead, we also have

$$
\left(\varepsilon+i \eta-\varepsilon_{1}\right) c_{1}-t_{0} c_{2}=1 .
$$

From Eqs. (A2A6), we get

$$
g_{0}= \begin{cases}\frac{\varepsilon+i \eta-\varepsilon_{2}}{(1+\lambda) t_{0}^{2}} & (\mathrm{VB}) \\ \frac{\varepsilon+i \eta-\varepsilon_{1}}{(1+\lambda) t_{0}^{2}} & (\mathrm{CB})\end{cases}
$$

$|\lambda| \geq 1$ is one of the roots of Eq. (A4). The surface Green's function of the right lead is identical.

We can also alternate the atom masses to generate a phonon band-gap. In our model the mass change will modify the renormalized spring constants. The diagonal elements of the dynamical matrix will be two alternating values $K_{i i}^{\alpha}=2 k_{1}$ or $2 k_{2}$, while the off-diagonal elements will be a single value $K_{i j}^{\alpha}=-\sqrt{k_{1} k_{2}}$, where $|i-j|=1$. If we assume that $k_{2} \geq k_{1}$, the acoustic band (AB) is $0<\omega^{2}<2 k_{1}$, and the optical band (OB) $2 k_{2}<\omega^{2}<$ $2\left(k_{1}+k_{2}\right)$. The surface Green's function is

$$
d_{0}= \begin{cases}\frac{\Omega_{2}}{(1+\lambda) k_{1} k_{2}} & (\mathrm{AB}) \\ \frac{\Omega_{1}}{(1+\lambda) k_{1} k_{2}} & (\mathrm{OB})\end{cases}
$$

where $\Omega_{n}=(\omega+i \eta)^{2}-2 k_{n} \cdot|\lambda| \geq 1$ is one of the roots of

$$
\lambda^{2}+\left(2-\frac{\Omega_{1} \Omega_{2}}{k_{1} k_{2}}\right) \lambda+1=0
$$

In all the simulation results of present paper, the two spring constants are equal $\left(k_{1}=k_{2}\right)$, which correspond a single continuum phonon band. The electron onsite energies are also equal $\left(\varepsilon_{1}=\varepsilon_{2}\right)$ except in Fig. 17, where we set $\varepsilon_{1}=-0.2 \mathrm{eV}$ and $\varepsilon_{2}=-0.1 \mathrm{eV}$ to mimic a semiconductor lead.

\section{APPENDIX B: ENERGY CURRENT CONSERVATION}

In this Appendix, we justify that the SCBA satisfies the energy current conservation. The justification of the electrical current conservation is given in the Refs $\stackrel{40,41}{.}$ What we need to prove is that

$$
\sum_{\alpha}\left(J_{\alpha}^{\mathrm{E}, \mathrm{e}}+J_{\alpha}^{\mathrm{E}, \mathrm{ph}}\right)=0 .
$$

The electron part is

$$
\sum_{\alpha} J_{\alpha}^{\mathrm{E}, \mathrm{e}}=\sum_{\alpha} \int \frac{d \varepsilon}{2 \pi} \varepsilon \operatorname{Tr}\left\{G^{>}(\varepsilon) \Sigma_{\alpha}^{<}(\varepsilon)-G^{<}(\varepsilon) \Sigma_{\alpha}^{>}(\varepsilon)\right\} .
$$

Using the important relation 39,40

$$
\operatorname{Tr}\left\{G^{>} \Sigma_{t}^{<}-G^{<} \Sigma_{t}^{>}\right\}=0,
$$

we get

$$
\sum_{\alpha} J_{\alpha}^{\mathrm{E}, \mathrm{e}}=-\int \frac{d \varepsilon}{2 \pi} \varepsilon \operatorname{Tr}\left\{G^{>}(\varepsilon) \Sigma_{\mathrm{eph}}^{<}(\varepsilon)-G^{<}(\varepsilon) \Sigma_{\mathrm{eph}}^{>}(\varepsilon)\right\} .
$$

The Hartree term does not contribute to the current directly. It's just like a static potential which only modifies the Green's function. Putting the Fock self-energy into Eq. (B4), we have

$$
\begin{aligned}
-Q= & \sum_{\alpha} J_{\alpha}^{E, e} \\
= & -i \int \frac{d \varepsilon}{2 \pi} \int \frac{d \omega}{2 \pi} \varepsilon\left[G_{n m}^{>}(\varepsilon) M_{m i}^{k} D_{k l}^{<}(\omega) G_{i j}^{<}(\varepsilon-\omega) M_{j n}^{l}\right. \\
& \left.-G_{n m}^{<}(\varepsilon) M_{m i}^{k} D_{k l}^{>}(\omega) G_{i j}^{>}(\varepsilon-\omega) M_{j n}^{l}\right] .
\end{aligned}
$$

Sum over all the indices is assumed. The heat generation $Q$ is the energy decrease of the electron system, which should also be the energy increase of the phonon system. Replacing $\omega$ by $-\omega$, using the symmetric properties of the phonon Green's functions $\frac{17}{}$, replacing $\varepsilon$ by $\varepsilon-\omega$, and finally changing dummy variables, we get

$$
\begin{aligned}
& i \int \frac{d \varepsilon}{2 \pi} \int \frac{d \omega}{2 \pi} \varepsilon\left[G_{n m}^{<}(\varepsilon) M_{m i}^{k} D_{k l}^{>}(\omega) G_{i j}^{>}(\varepsilon-\omega) M_{j n}^{l}\right] \\
= & i \int \frac{d \varepsilon}{2 \pi} \int \frac{d \omega}{2 \pi} \varepsilon\left[G_{n m}^{<}(\varepsilon) M_{m i}^{k} D_{k l}^{>}(-\omega) G_{i j}^{>}(\varepsilon+\omega) M_{j n}^{l}\right] \\
= & i \int \frac{d \varepsilon}{2 \pi} \int \frac{d \omega}{2 \pi}(\varepsilon-\omega)\left[G_{n m}^{<}(\varepsilon-\omega) M_{m i}^{k} D_{l k}^{<}(\omega) G_{i j}^{>}(\varepsilon) M_{j n}^{l}\right] \\
= & i \int \frac{d \varepsilon}{2 \pi} \int \frac{d \omega}{2 \pi}(\varepsilon-\omega)\left[G_{n m}^{>}(\varepsilon) M_{m i}^{k} D_{k l}^{<}(\omega) G_{i j}^{<}(\varepsilon-\omega) M_{j n}^{l}\right] .
\end{aligned}
$$

Putting Eq. (B6) back into Eq. (B5), we get

$$
\begin{aligned}
-Q & =\sum_{\alpha} J_{\alpha}^{\mathrm{E}, \mathrm{e}} \\
& =-i \int \frac{d \varepsilon}{2 \pi} \int \frac{d \omega}{2 \pi} \omega\left[G_{n m}^{>}(\varepsilon) M_{m i}^{k} D_{k l}^{<}(\omega) G_{i j}^{<}(\varepsilon-\omega) M_{j n}^{l}\right] \\
& \neq 0 .
\end{aligned}
$$

For the phonon energy current we have

$$
\begin{aligned}
Q= & \sum_{\alpha} J_{\alpha}^{\mathrm{E}, \mathrm{ph}} \\
= & i \int \frac{d \varepsilon}{2 \pi} \int \frac{d \omega}{4 \pi} \omega\left[D_{n m}^{>}(\omega) M_{l k}^{m} G_{k i}^{<}(\varepsilon) G_{j l}^{>}(\varepsilon-\omega) M_{i j}^{n}\right. \\
& \left.-D_{n m}^{<}(\omega) M_{l k}^{m} G_{k i}^{>}(\varepsilon) G_{j l}^{<}(\varepsilon-\omega) M_{i j}^{n}\right] .
\end{aligned}
$$


Following the same procedure as electrons, finally we get

$$
\begin{aligned}
Q & =\sum_{\alpha} J_{\alpha}^{\mathrm{E}, \mathrm{ph}} \\
& =i \int \frac{d \varepsilon}{2 \pi} \int \frac{d \omega}{2 \pi} \omega\left[G_{n m}^{>}(\varepsilon) M_{m i}^{k} D_{k l}^{<}(\omega) G_{i j}^{<}(\varepsilon-\omega) M_{j n}^{l}\right] \\
& \neq 0 .
\end{aligned}
$$

So we still have

$$
\sum_{\alpha}\left(J_{\alpha}^{\mathrm{E}, \mathrm{e}}+J_{\alpha}^{\mathrm{E}, \mathrm{ph}}\right)=0 .
$$

Eqs. B7. B9 give the energy exchange between the electron and the phonon system, which is also the heat generation of the atomic junction. Replacing $D^{<}, G^{<}$ by $D_{0}^{<}, G_{0}^{<}$in Eq. (B7), and $G^{>}, G^{<}$by $G_{0}^{>}, G_{0}^{<}$in Eq. (B9), we get the results under BA. We can find that the energy increase of the phonons does not equal to the energy decrease of the electrons under BA.

\section{APPENDIX C: ANALYTICAL RESULT AT ZERO TEMPERATURE}

At zero temperature, we can get an analytical expression for heat generation in a single-atom structure by using the bare Green's functions in Eq. (B7] B9). We only take into account the imaginary part of the lead selfenergies and ignore their energy dependence (the wideband limit) ${ }^{24}$. Finally, we assume that the phonons are in their equilibrium states. Under these approximations, the heat generation is (assuming $e V \geq \omega_{0}$ )

$$
\begin{aligned}
Q \approx & \frac{1}{2} M^{2} \Gamma_{L} \Gamma_{R} \int_{-\frac{e V}{2}+\omega_{0}}^{\frac{e V}{2}} \frac{d \varepsilon}{2 \pi} \\
& \times \frac{1}{\left[\left(\varepsilon-\varepsilon_{0}\right)^{2}+\Gamma^{2} / 4\right]\left[\left(\varepsilon-\varepsilon_{0}-\omega_{0}\right)^{2}+\Gamma^{2} / 4\right]} \\
= & \frac{M^{2} \Gamma_{L} \Gamma_{R}}{4 \pi\left(\omega_{0}^{2}+\Gamma^{2}\right)}\left\{\frac { 1 } { \omega _ { 0 } } \left[\ln \left(\frac{\left(e V / 2-\varepsilon_{0}\right)^{2}+\Gamma^{2} / 4}{\left(e V / 2-\varepsilon_{0}-\omega_{0}\right)^{2}+\Gamma^{2} / 4}\right)\right.\right. \\
& \left.-\ln \left(\frac{\left(-e V / 2-\varepsilon_{0}+\omega_{0}\right)^{2}+\Gamma^{2} / 4}{\left(-e V / 2-\varepsilon_{0}\right)^{2}+\Gamma^{2} / 4}\right)\right] \\
& +\frac{2}{\Gamma}\left[\arctan \left(\frac{e V / 2-\varepsilon_{0}}{\Gamma / 2}\right)+\arctan \left(\frac{e V / 2-\varepsilon_{0}-\omega_{0}}{\Gamma / 2}\right)\right. \\
& -\arctan \left(\frac{-e V / 2-\varepsilon_{0}}{\Gamma / 2}\right) \\
& \left.\left.-\arctan \left(\frac{-e V / 2-\varepsilon_{0}+\omega_{0}}{\Gamma / 2}\right)\right]\right\} .
\end{aligned}
$$

$\omega_{0}$ is the phonon energy, $\varepsilon_{0}$ is the electron onsite energy, $V$ is the applied bias, and $\Gamma=\Gamma_{L}+\Gamma_{R}$. The heat generation is zero when $\mathrm{eV} \leq \omega_{0}$. Equation (C1) can reproduce most the qualitative features of heat generation in a single atom, except that it does not take into account heat conduction in the phonon system.
* Electronic address: tower.lu@gmail.com

$\dagger$ http://staff.science.nus.edu.sg/ phywjs/

1 S. Ciraci, A. Buldum, and I. P. Batra, J. Phys.: Condens. Matter 13, R537 (2001).

2 B. J. van Wees, H. van Houten, C. W. J. Beenakker, J. G. Williamson, L. P. Kouwenhoven, D. van der Marel, and C. T. Foxon, Phys. Rev. Lett. 60, 848 (1988); D. A. Wharam, T. J. Thornton, R. Newbury, M. Pepper, H. Ahmed, J. E. F. Frost, D. G. Hasko, D. C. Peacock, D. A. Ritchie, and G. A. C. Jones, J. Phys. C: Solid State Phys. 21, L209 (1988).

${ }^{3}$ L. G. C. Rego and G. Kirczenow, Phys. Rev. Lett. 81, 232 (1998); K. Schwab, E. A. Henriksen, J. M. Worlock, and M. L. Roukes, Nature 404, 974 (2000).

4 E. Pop, D. Mann, J. Cao, Q. Wang, K. Goodson, and H. Dai, Phys. Rev. Lett. 95, 155505 (2005); D. Mann, Y. K. Kato, A. Kinkhabwala, E. Pop, J. Cao, X. Wang, L. Zhang, Q. Wang, J. Guo, and H. Dai, Nature Nanotechnology 2, 33 (2007).

5 M. Lazzeri, S. Piscanec, F. Mauri, A. C. Ferrari, and J. Robertson, Phys. Rev. Lett. 95, 236802 (2005).

${ }^{6}$ D. G. Cahill, W. K. Ford, K. E. Goodson, G. D. Mahan, A. Majumdar, H. J. Maris, R. Merlin, and S. R. Phillpot, J. Appl. Phys. 93, 793 (2003).

7 S. Lepri, R. Livi, and A. Politi, Phys. Rep. 377, 1 (2003).

8 B. Li, J. Wang, L. Wang, and G. Zhang, Chaos 15, 015121
(2005).

9 L. V. Keldysh, Sov. Phys. JETP 20, 1018 (1965).

10 L. Kadanoff and G. Baymn, Quantum Statistical Mechanics (W. A. Benjamin, New York, 1962).

11 H. Haug and A.-P. Jauho, Quantum Kinetics in Transport and Optics of Semiconductors (Springer, Berlin, 1996).

12 S. Datta, Electronic Transport in Mesoscopic Systems (Cambridge University Press, 1997).

13 A. Ozpineci and S. Ciraci, Phys. Rev. B 63, 125415 (2001).

14 N. Mingo and L. Yang, Phys. Rev. B 68, 245406 (2003); Phys. Rev. B 70, 249901(E) (2004); N. Mingo, Phys. Rev. B 74, 125402 (2006).

15 T. Yamamoto and K. Watanabe, Phys. Rev. Lett. 96, 255503 (2006).

16 A. Dhar and D. Sen, Phys. Rev. B 73, 085119 (2006).

17 J.-S. Wang, J. Wang, and N. Zeng, Phys. Rev. B 74, 033408 (2006); J.-S. Wang, N. Zeng, J. Wang, and C. K. Gan, cond-mat/0701164.

18 M. Galperin, M. A. Ratner, and A. Nitzan, J. Phys.: Condens. Matter 19, 103201 (2007); cond-mat/0611169.

19 D. A. Ryndyk, M. Hartung, and G. Cuniberti, Phys. Rev. B 73, 045420 (2006).

20 C. Auer, F. Schurrer, and C. Ertler, Phys. Rev. B 74, 165409 (2006).

21 M. Lazzeri and F. Mauri, Phys. Rev. B 73, 165419 (2006).

22 C. Caroli, R. Combescot, P. Nozieres, and D. Saint-James, 
J. Phys. C : Solid State Phys. 4, 916 (1971).

23 We denote electron energy as $\varepsilon$, and phonon energy as $\omega$, except in the unified expressions including both electrons and phonons, i.e., Eq. (22).

${ }^{24}$ Y. Meir and N. S. Wingreen, Phys. Rev. Lett. 68, 2512 (1992); A.-P. Jauho, N. S. Wingreen, and Y. Meir, Phys. Rev. B 50, 5528 (1994).

25 R. Lake and S. Datta, Phys. Rev. B 46, 4757 (1992).

26 T. N. Todorov, Phil. Mag. B 77, 965 (1998).

27 M. J. Montgomery, T. N. Todorov, and A. P. Sutton, J. Phys.: Condens. Matter 14, 5377 (2002).

28 A. P. Horsfield, D. R. Bowler, H. Ness, C. G. Snchez, T. N. Todorov, and A. J. Fisher, Rep. Prog. Phys. 69, 1195 (2006).

29 N. Agraït, C. Untiedt, G. Rubio-Bollinger, and S. Vieira, Phys. Rev. Lett. 88, 216803 (2002).

30 A. Pecchia, G. Romano, and A. D. Carlo, Phys. Rev. B 75, 035401 (2007).

31 Q.-F. Sun and X. C. Xie, cond-mat/0608536.

32 T. N. Todorov, J. Hoekstra, and A. P. Sutton, Phys. Rev. Lett. 86, 3606 (2001).

${ }^{33}$ M. D. Ventra, Y.-C. Chen, and T. N. Todorov, Phys. Rev. Lett. 92, 176803 (2004).
34 Y.-C. Chen, M. Zwolak, and M. D. Ventra, Nano Lett. 3, 1691 (2003); Z. Yang, M. Chshiev, M. Zwolak, Y.-C. Chen, and M. D. Ventra, Phys. Rev. B 71, 041402(R) (2005); Z. Huang, B. Xu, Y. Chen, M. D. Ventra, and N. Tao, Nano Lett. 6, 1240 (2006).

35 D. Segal and A. Nitzan, J. Chem. Phys. 117, 3915 (2002).

${ }^{36}$ N. Guisinger, M. Greene, R. Basu, A. Baluch, and M. Hersam, Nano Lett. 4, 55 (2004).

37 T. Rakshit, G.-C. Liang, A. W. Ghosh, M. C. Hersam, and S. Datta, Phys. Rev. B 72, 125305 (2005).

38 J. Taylor, H. Guo, and J. Wang, Phys. Rev. B 63, 245407 (2001); N. Sergueev, D. Roubtsov, and H. Guo, Phys. Rev. Lett. 95, 146803 (2005).

39 T. Frederiksen, M. Brandbyge, N. Lorente, and A.-P. Jauho, Phys. Rev. Lett. 93, 256601 (2004); T. Frederiksen, M. Paulsson, M. Brandbyge, and A.-P. Jauho, condmat/0611562.

40 T. Frederiksen, Master's thesis, Technical University of Denmark (2004).

41 J. K. Viljas, J. C. Cuevas, F. Pauly, and M. Hafner, Phys. Rev. B 72, 245415 (2005). 